\title{
The Research on Planning the Containers' Concentration and Untwining for Container Storage Station of Port Enterprise
}

\author{
Ting He, Xiaohong Dong and Hanchuan Xu \\ School of Computer Science and Technology, Harbin Institute of Technology, Harbin, Heilongjiang, China \\ \{xuantinghe \& xhc\}@ hit.edu.cn
}

\begin{abstract}
Aiming at improving the service production efficiency and service level of port enterprises effectively, a planning and optimization model with the characteristics of multi-objective and discrete value domain is developed for the containers' concentration and untwining between container storage station and harbor. Then a dynamic, adaptive genetic algorithm proposed is used to solve the above model. Finally, the researches of the model and algorithm proposed are also validated through a case study.
\end{abstract}

Keywords - container storage station, containers' concentration and untwining, planning model; dynamic and adaptive genetic algorithm

\section{港口企业场站集装箱集港/疏港计划优化问题研究}

\author{
何霆 董晓红 徐汉川 \\ 哈尔滨工业大学计算机科学与技术学院，哈尔滨，中国
}

\begin{abstract}
摘 要 为了有效地提高港口企业的服务生产效率和服务水平, 本文以港口企业场站一码头间的集装箱集港/疏港计划问题为研究 对象, 建立了具有多目标和离散值域特点的场站集装箱集港/疏港计划优化模型, 并针对此问题设计了动态自适应实数加速遗传算法, 并对该模型进行了求解。最后，通过实验验证了所建立模型、所提算法的合理性和有效性。
\end{abstract}

关键词 格式要求, 集装箱的集港/疏港, 计划优化模型, 动态自适应遗传算法

\section{1. 引言}

伴随着全球经济一体化, 世界经济的持续发展促使各 国之间货物贸易往来日趋频繁。众多的海运物流企业群通 过对全球物流链条上相关企业的物流服务资源进行了有效 组合与集成, 实现了世界海运物流领域的持续发展, 为全 球经济的不断发展提供了坚实的物质基础, 并产生了巨大 的经济效益和社会效益 $[1]$ 。

在海运物流系统中, 港口企业作为物质中转节点, 是 整个物流链上下游环节的中枢。长期以来, 港口企业是否 可以有效的组织已有的物流资源并且提高其服务水平和服 务效率是港口企业亟待解决的问题 [2]。而在港口企业中,

国家科技部 863 计划和国家自然科学基金项目支持 (资助号: 2012AA040904 和 71171066)
其场站的集装箱集港/疏港计划水平则是直接影响港口企 业运转效率高低和服务水平的关键因素, 因为它直接影响 到上下游环节的再运输。因此, 在对国内外相关研究进行 综合分析的基础上，基于场站的各种物流资源，本文以场 站集装箱集港/疏港物流设备负载均衡为约束, 缩短了港口 周转时间为目标, 开展了本研究。

\section{2. 国内外相关研究}

场站是海运物流供应链的核心环节, 场站集装箱的集 港/疏港能力更是影响场站周转效率的主要因素, 为了有效 的提高场站的生产效率和服务水平, 国内外学者对此相关 领域进行了大量的研究工作。

针对场站集装箱的集港/疏港研究问题, Avriel $\mathrm{M}$ 以最 
小化吊装费用为目标, 研究了集装箱装卸序列调度优化问 题, 通过分析指出此问题是个 NP 问题, 并指出其与图着 色问题间的对应关系, 从而提出一个基于图着色问题的启 发式规则[3]。Ryu K. R. 通过采用基于 Tabu 搜索算法及 蚂蚁算法的启发式过程对集装箱装载过程进行调度的优 化, 并对这两种算法的计算效率进行了比较 [4]。Richard Lima 研究了几个工班的龙门吊的配置问题, 文中给出了一 个两阶段模型, 首先考虑堆场机械的作业均衡, 将到达集 装箱按类型分配到箱区, 然后再考虑最大限度地降低翻箱 率, 将集装箱具体放到某一个位置。但是在每个工班之内 的移动次数不能超过 1 次, 该假设与实际情况存在较大的 差距 ${ }^{[5]}$ 。W.C Ng 通过在混合整数规划方法的基础上运用分 支定界算法, 为堆场起重机解决了在其运动区域范围内对 不同时间段对集装箱装卸的调度问题, 通过该堆场起重机 工作调度表，有效地减少了工作等待时间[6]。

上可以看出, 当前已有的一些研究大多是针对场站集 装箱集港/疏港的单一目标进行研究, 缺乏从场站一码头之 间集装箱转运的物流服务资源和负荷均衡角度出发的研究 成果。基于此, 本文试图以所承担的某港口企业场站集装 箱集港疏港计划调度项目为支撑, 通过细致的调查研究, 提供此类问题的解决方案。

\section{3. 场站集装箱集港/疏港计划优化模型}

港口集装箱集港业务过程指货物由码头后方场站通过 龙门吊设备和车队装卸搬运到达码头前方场站等待装船的 过程。港口集装箱疏港业务过程指船舶卸货完毕后货物由 码头前方场站通过龙门吊设备和车队装卸搬运到达码头后 方场站的过程。在建立模型之前, 首先了解一下对场站集 装箱集疏港所涉及的一些术语。

场站及堆存空间: 场站是由称作箱区的长方形区域组 成的 (如图 1), 沿着箱区长边一个 TEU 的长度称作贝 (bay, 场站纵列位置), 沿着箱区短边一个 TEU 的宽度称列位 (row, 场站横行位置), 贝当中的一排称作垛, 垛的坚直 方向有很多层 (tier), 每垛当中的一层称作一个箱位, 箱 位是堆场的最小存储单元。集装箱在场站当中按照 “并列 方式” 进行排列, 行之间空有固定距离方便装卸设备对集 装箱进行操作。

集装箱装卸服务能力直接制约着其他服务能力的表 现, 如果没有合理的场站装卸和运输服务设备调度, 就会 使集卡之间产生拥堵问题 (水平运输服务能力), 使岸桥不 能合理的得到利用 (码头装卸服务能力), 船舶的在港等待 时间增长。而一般装卸设备对集装箱是按先来先服务的原 则进行工作的, 虽然能够保证在规定时间段内完成对集装
箱的装卸操作任务, 但是可能由于龙门吊工作量的不均衡, 导致集卡频繁出入于同一地点, 出现拥堵, 使码头岸桥得 不到有效的利用, 延长船舶在港等待时间, 使海运物流服 务效率低下。所以有效的解决办法就是对场站装卸运输设 备的服务能力和负荷进行合理的计划优化, 从而提高装卸 运输服务效率和水平。

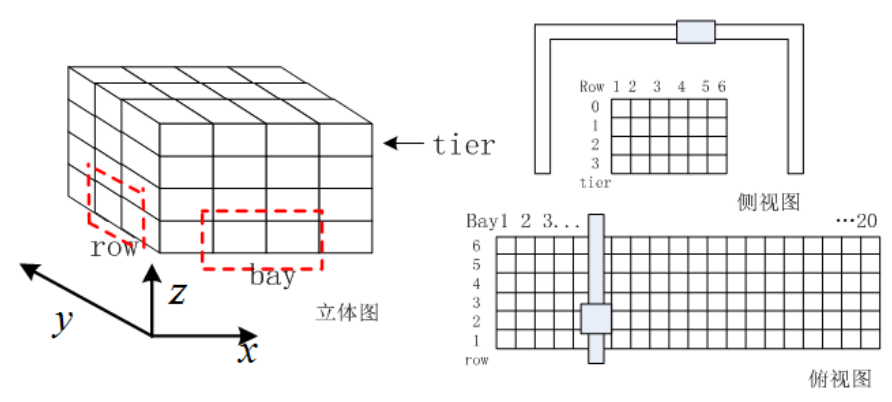

图 1 场站堆区示意图

在集装箱的整个物流服务过程当中, 场站不仅作为水 路和陆路运输的连接点, 更是集装箱多式联运的枢纽, 具 有多种运输方式转化的功能, 在集装箱集港/疏港的过程当 起到缓冲的作用。场站集装箱集港/疏港计划模型的建立, 为保证集装箱在集港/疏港过程可以高度严密的进行流水 作业提供了保证。场站的空间资源、装卸资源、运输资源 为计划模型的建立提供了物质基础。

集装箱集港过程中船舶按照码头预先发出的指令进行 指定泊位的停靠, 每个泊位对应场站当中的不同堆区, 采 取不同的堆存/装载策略。在集装箱疏港过程进行集装箱出 口操作时, 从船舶停靠泊位对应的场站堆区将集装箱运送 到该泊位, 当进行集装箱进口操作时, 从船舶停靠的泊位 将集装箱运送到该泊位所对应场站的堆区进行集装箱的堆 存。合理安排堆场资源, 不仅能够减少岸桥的等箱时间, 减少翻箱率, 提高堆场装卸运输速度, 而且可以大幅度提 高集装箱的流通效率, 缩短集装箱周转周期, 提高服务能 力的输出。

本节以集装箱周转效率最高、最小化龙门吊的最大作 业时间, 缩短集卡的运输时间为目标, 建立了集装箱集港/ 疏港计划优化模型。

\section{(1) 条件假设}

1) 已知所需进行操作集装箱在场站中的堆存位置。

2) 龙门吊为吊起集装箱而对其上锁的时间与为放下该 集装箱所用的解锁时间相等;

3) 进行操作时, 忽略为了对压在下面的集装箱进行操 作而将上面的集装箱挪动到相邻箱位所用的时间; 
4）场站内集卡运送集装箱的路径是优化后的最佳运送 路径。

(2) 目标函数

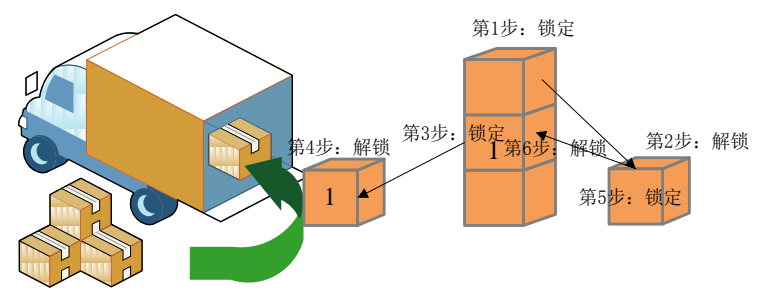

图 2 为取 1 号箱进行的翻箱操作

$$
\operatorname{Min}\left(\operatorname{Max}_{m_{j}} \sum_{\left\{i \mid m_{i}=m_{j}\right\}}\left(T_{i}^{s}+T_{i}^{c}\right)\right)
$$

式中:

$$
\begin{gathered}
T_{i}^{s}=\left\{\begin{array}{c}
T_{1}^{m}+T_{2}^{m}+T, h_{i t}=0 \\
h_{i t} \times 2 \times\left(T_{1}^{m}+T_{2}^{m}+T\right)+\left(T_{1}^{m}+T_{2}^{m}+T\right), h_{i t} \neq 0
\end{array}\right. \\
T_{i}^{c}=\frac{x_{i} \times l}{v_{1}^{m}}+\frac{y_{i} \times w}{v_{2}^{m}}+\frac{s_{i}}{v}
\end{gathered}
$$

约束条件

$\forall i \neq j$, 如果 $x_{i}=x_{j}, y_{i}=y_{j}$, 则 $h_{i t}=h_{j t}$;

$\forall i \neq j$, 如果 $m_{i}=m_{j}$, 则 $t_{i}=t_{j}$;

$\forall i \neq j, t_{i}<t_{j}$, 如果 $x_{i}=x_{j}, y_{i}=y_{j}, h_{i t}<h_{j t}$, 则 $h_{i t}=h_{j t}-1$

(3) 符号说明

$i, j$ : 集装箱或龙门吊对应编号下标;

$n:$ 龙门吊的个数;

$N$ : 周期内所需操作的集装箱数量;

$m_{i}, m_{j}$ ：对第 $i / j$ 号集装箱进行装卸操作的龙门吊;

$T_{i}^{s}$ : 龙门吊到达所需操作 $i$ 号集装箱所需时间;

$T_{i}^{c}$ : 集卡将集装箱 $i$ 从所在箱区运输到船舶停靠码头 泊位的时间;

$x_{i}$ : 集装箱 $i$ 所在箱区的行号;

$y_{j}$ : 集装箱 $i$ 所在箱区的列号;

$h_{i t}$ : 在 $t$ 时段里集装箱 $i$ 所在箱区的层号;

$l:$ 场站箱区一列的长度;

$w$ : 场站箱区一行的长度;

$s_{i}$ : 集卡从集装箱 $i$ 所在箱区运输到船舶停靠的码头泊 位的水平距离;

$T_{1}^{m}$ ：为了吊起集装箱而对集装箱上锁的时间;
$T_{2}^{m}$ : 为了放下集装箱而对集装箱解锁的时间;

$T$ : 所要操作的集装箱不在最上面, 为了对集装箱进 行操作, 在翻箱时移动上层集装箱到指定位置时所需时间;

$v_{1}^{m}:$ 龙门吊大车的移动速度;

$v_{2}^{m}:$ 龙门吊小车的移动速度;

$v$ : 集卡的水平运输速度;

（4）模型说明

1) 式是使船舶在港滞留时间最短的目标函数。船舶在 港的等待时间主要取决于要装船的所有集装箱到达船舶所 在泊位的时间, 而这些集装箱的装卸任务平分给场站的龙 门吊进行操作并由集卡运输到船舶所在的泊位, 所以工作 时间最长的龙门吊影响船舶在港时间, 本目标通过使作业 时间最长的龙门吊的时间最短来实现缩短船舶在港滞留时 间目标。目标函数由两部分组成, 集装箱装卸集装箱时间 和集卡运输时间。

2) 式是集装箱装卸时间的表达式。当所需操作的集装 箱位于最上层时无需进行翻箱操作, 时间就是从集装箱所 在位置放到集卡上所需的时间。当所需操作的集装箱被压 在下面时, 需要进行翻箱操作, 时间是翻箱操作时间加上 将所需操作集装箱放上集卡两部分时间之和。

3 ) 式是龙门吊大车和小车到达所需进行操作集装箱所 需时间和集卡将所需进行操作的集装箱从其所在箱区运输 到船舶所在泊位三部分时间之和。

4）式保证在同一个箱位只能存放一个集装箱。

5) 式保证在同一时段,一个龙门吊只能为一个集装箱 进行装卸操作。

6）式保证当已经对上层的集装箱完成了装卸任务, 其 下层在此之后需要进行装卸的集装箱的层数应该减少一 层。

\section{4. 模型求解的动态自适应遗传算法}

本文建立的场站集装箱集港/疏港计划优化模型具有 动态、离散值域、多约束条件等特征。标准的遗传算法具 有很强的全局寻优搜索能力, 但随着问题规模的增长, 标 准遗传算法很难保证算法的收玫速度。为了对模型进行有 效的求解, 本文在标准遗传算法研究的基础之上, 结合模 型所具有的特征提出了针对本模型改进的遗传算法。如下:

(1) 为了避免传统遗传算法在进化当中出现的停止不 前现象, 满足动态、自适应的进行交叉和变异过程, 本文 受神经网络当中经常使用的神经元激活函数 signmoid 函数 的启发, 本文将交叉率 $p_{c}$ 和变异率 $p_{m}$ 设计为 


$$
\begin{aligned}
& p_{c}=\left\{\begin{array}{lr}
\frac{p_{c \max }-p_{c \min }}{1+\exp \left(\frac{\left(f^{\prime}-f_{\text {avg }}\right)}{f_{\text {max }}-f_{\text {avg }}}\right)}+p_{c \min }, & f^{\prime} \geq f_{\text {avg }} \\
p_{c \max } & , f^{\prime}<f_{\text {avg }}
\end{array}\right. \\
& p_{m}=\left\{\begin{array}{lr}
\frac{p_{m \max }-p_{m \min }}{1+\exp \left(\frac{\left(f-f_{\text {avg }}\right)}{f_{\text {max }}-f_{\text {avg }}}\right)}+p_{\text {min }}, & f \geq f_{\text {avg }} \\
p_{m \text { max }} & , f<f_{\text {avg }}
\end{array}\right.
\end{aligned}
$$

其中 $f_{\text {max }}$ 表示种群的最大适应度, $f_{\text {avg }}$ 表示种群的平均 适应度, $f^{\prime}$ 表示参与交叉的两个个体中较大的适应度, $f$ 表示变异个体的适应度。通过对交叉算子和变异算子的改 进可以解决动态自适应的问题。

(2) 针对所建立模型具有离散值域的特点本文采用自 然数进行编码, 染色体当中的各个值代表的变量的取值对 应其在离散集合当中的序号。使用自然数进行编码具有搜 索空间小, 操作简单, 直观的特点, 可提高问题求解速度。

基于改进遗传算法对模型的求解过程如下。

(1) $C$ : 个体编码方法。对所需装卸的集装箱进行自 然数编码, 染色体长度为所需装卸集装箱数量, 在相同的 染色体当中不可以重复出现同一基因代码 (集装箱)。而染 色体串中基因代码的顺序就是集装箱的操作顺序。按照龙 门吊数量可以将染色体串拆分为几个染色体片段 (染色体 片段个数等于龙门吊个数), 不同的染色体片段代表不同龙 门吊对集装箱的操作序列。

(2) $E$ : 个体适应度评价函数。选取目标函数作为适 应度函数, 因为该目标函数为一个最小化问题, 个体通过 适应度值的大小进行保留或者淘汰操作, 适应度值越小说 明个体的适应性越强, 适应度值越大说明适应度越差, 保 留适应度强的个体淘汰适应度差的个体。

(3) $P_{0}$ : 初始种群。利用港口历史数据, 采用启发式 策略初始种群。

(4) $M$ : 种群大小。种群大小选择为 20 。

（5） $\Phi$ : 选择算子。本文选择各代当中适应度最高的 两个个体直接进入到下一代当中。

（6） $\Gamma$ : 交叉算子。本文采用采用动态自适应公式 (1) 产生单点交叉。受到模型条件的约束, 通过此种交叉方式 可能会产生非正常个体。通过对所有子代同时进行如下操 作使其转换为正常的子个体:

首先, 从左到右依次扫描个体, 记录出现重复基因代 码的位置及重复的基因代码, 最后得到正常的子个体。

（7） $\Psi$ : 变异算子。本文采用交换位的变异方法, 即 动态自适应根据公式 (2) 产生 1 到染色体串长度之间的两
个随机数, 将这两个位置上的基因代码进行交换, 因为在 一个染色体串当中不会出现相同的基因代码所以变异以后 仍然是正常的个体, 而且可以保证集装箱的操作顺序进行 了改变, 保证了种群的多样性, 例: 子代 1:3457126 随 机的两个随机数为 3,5 则变异后的个体为子代 1': 3417 526 。

（8） T: 遗传算法终止条件。本为设置的终止条件是 进行进化 100 代。

\section{4. 模型和算法验证分析}

某港口企业是中国东部沿海的一家大型海运物流服务 企业, 其集装箱码头采用桥吊-集卡-龙门吊装卸工艺进行集 港疏港作业。其拥有 6 个集装箱码头, 每个码头配有两个 泊位, 每个泊位与其场站当中的 6 个箱区相对应, 每个箱 区有 20 贝、 6 列, 龙门吊最大可操作层数为 5 层。考虑到 翻箱操作, 预留出每垛最上一层为空, 所以每垛最大层数 为 4 层。根据码头历史数据, 泊位中每艘船平均每天要对 500 个集装箱进行出口操作。集装箱集疏港计划优化模型 的输入信息, 如下表 1 和表 2 所示。

\section{表 1 模型的输入数据}

\begin{tabular}{|c|l|c|}
\hline 符号 & \multicolumn{1}{|c|}{ 符号在模型中的物理含义 } & 数值 \\
\hline$N$ & 龙门吊个数 & 10 台 \\
\hline$N$ & 周期内所需操作的集装箱数量 & 500 箱 \\
\hline$l$ & 场站箱区一列的长度; & $2.653 \mathrm{~m}$ \\
\hline$w$ & 场站箱区一行的长度; & $6.379 \mathrm{~m}$ \\
\hline$v_{1}^{m}$ & 龙门吊大车移动速度; & $0.417 \mathrm{~m} / \mathrm{s}$ \\
\hline$v_{2}^{m}$ & 龙门吊小车移动速度; & $4.167 \mathrm{~m} / \mathrm{s}$ \\
\hline$v$ & 集卡水平运输速度; & $15 \mathrm{~m} / \mathrm{s}$ \\
\hline$T_{1}^{m}$ & 吊起集装箱所需集装箱上锁时间 \\
\hline$T_{2}^{m}$ & 放下集装箱所需集装箱解锁时间 & $15 \mathrm{~s}$ \\
\hline$T$ & $\begin{array}{l}\text { 翻箱时移动上层集装箱到指定位置 } \\
\text { 所需时间 }\end{array}$ & $0 \mathrm{~s}$ \\
\hline
\end{tabular}

表 2 泊位到各箱区的水平距离

\begin{tabular}{|l|l|l|l|l|l|l|}
\hline & 箱区 1 & 箱区 2 & 箱区 3 & 箱区 4 & 箱区 5 & 箱区 6 \\
\hline 泊位箱区距离 & $125 \mathrm{~m}$ & $175 \mathrm{~m}$ & $225 \mathrm{~m}$ & $275 \mathrm{~m}$ & $325 \mathrm{~m}$ & $375 \mathrm{~m}$ \\
\hline
\end{tabular}

(2) 模型求解结果分析

基于本文所提模型和算法, 对上述作业任务进行计划 优化。同时, 为了进行算法运算结果的分析, 本文分别采 取了不具备自适应求解操作的标准遗传算法和本文算法两 种计算策略进行求解。求解结果如下图 3、图 4。 


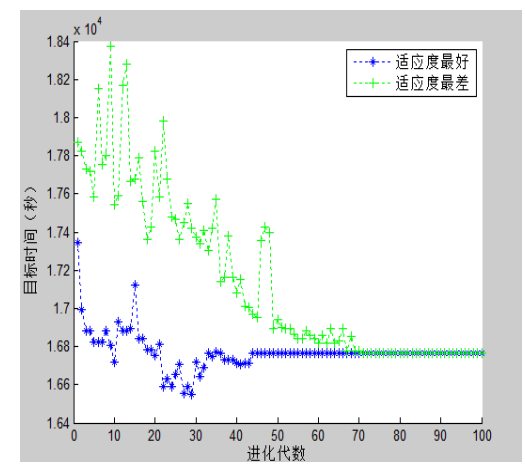

图 3 标准遗传算法和本文算法求解结果收敛速度对比图
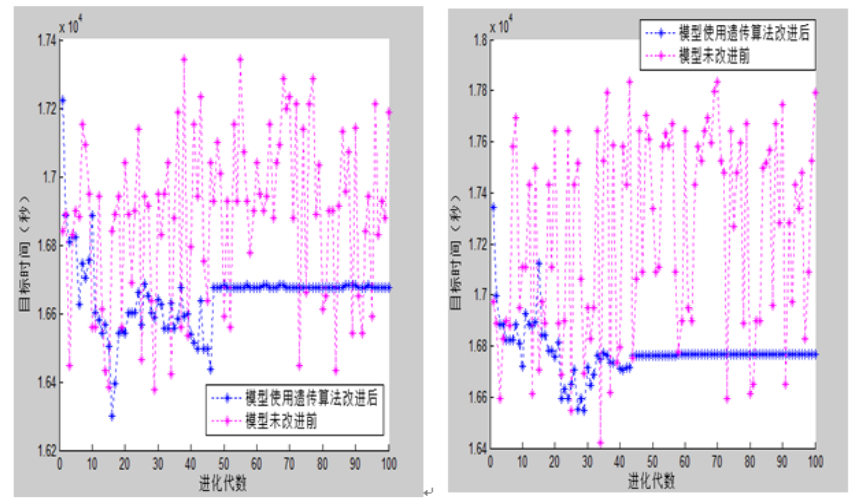

图 4 模型求解收敛速度和求解结果对比

在使用了本文提出的交叉率 $p_{c}$ 和变异率 $p_{m}$ 的条件下保 证了算法自适应的进行交叉和变异, 通过观察输出结果可 以发现本文提出改进的遗传算法在 45 代左右就已经收敛 了, 而未使用之前需要 70 代才可以收玫, 加快了收玫速度, 并且通过对比本文提出的遗传算法和未使用之前可以发现 算法对模型目标的优化效果明显优于算法未改进之前, 而 且优化的效果也比较明显。

表 3 改进遗传算法和未改进遗传算法求解结果对比表

\begin{tabular}{|c|c|c|c|c|c|}
\hline 计算代数 & 5 & 10 & 20 & 30 & 40 \\
\hline 改进前时间 & 1.773 & 1.760 & 1.756 & 1.743 & 1.712 \\
\hline 改进后时间 $)$ & 1.685 & 1.681 & 1.676 & 1.642 & 1.662 \\
\hline
\end{tabular}

考虑到集装箱装船的顺序, 减少装船时集装箱的翻箱 率, 可以在船舶待港所允许的时间内将需要装船的集装箱 分时段分别运抵船舶等待泊位, 通过图 4 表明, 该算法都 可以适应所划分时段，对龙门吊作业时间进行优化。

通过输出结果可以看出在相同装卸策略、相同场站资 源条件下, 使用本文遗传算法对所提出的模型进行了有效 的改进, 缩短了船舶在港等待时间, 加快了船舶周转速度。
6. 结论

港口企业场站物流服务资源的合理利用，对于港口企 业提高服务水平, 提升服务效率, 降低物流服务成本具有 重要的意义。本文以港口企业场站的堆存资源、装卸资源 和运输资源为基础, 结合某企业提升物流服务效率的实际 需求, 以合理的编排场站装卸设备、运输设备资源生产任 务、缩短港口集装箱周转时间为目的, 建立了场站集装箱 集港/疏港计划模型, 提出了相关的模型求解算法。本文的 主要贡献可以概括为以下几点：立足于港口企业如何提高 服务输出能力, 建立了场站集装箱集港/疏港计划优化模 型, 充分考虑了场站的实际物流资源和物流负荷之间的均 衡调度; 针对所建立的优化模型及特点, 设计了求解上述 模型的动态自适应遗传算法。最后, 通过实例验证了所见 模型和算法的有效性。

\section{参考文献(References)}

[1] T.W. Lee, N.K. Park and D.W. Lee, "A simulation study for the logistics planning of a container terminal in view of SCM," Maritime Policy \& Management, vol.30, pp. 243-254, 2003.

[2] W. C. Ng and K. L. Mak, "Yard crane scheduling in port container terminals," Applied Mathematical Modelling, vol.29, pp.263-276, 2005.

[3] M. Avriel, M. Penn and N. Shpirer, "Container ship stowage problem: complexity and connection to the coloring of circle graphs," Discrete Applied Mathematics, vol. 103, pp.271-279, 2000.

[4] K. R. Ryu, K. H. Kim and Y. H. Lee, "Loading Sequencing Algorithms for container Ships by Using Metaheuristics," 16th International Conference On Production Research, Prague, Czech Republic, August 2001.

[5] R. Lima and C. Zhang, "A heuristic for dynamic yard crane deployment in a container terminal," IIE Transactions, vol. 35, pp. 161-174, 2003.

[6] A. C. Paixão and P. B. Marlow, "Fourth generation ports - a question of agility?," International Journal of Physical Distribution \& Logistics, vol. 33, pp. 355-376, 2003.

[7] A. Imai, E. Nishimura and S. Papadimitriou, "Berth allocation with service priority," Transportation Research Part B: Methodological, vol. 37, pp. 437-457, 2003.

[8] D. E. Goldberg, "Genetic Algorithms in Search, Optimization, and Machine Learning," Addison -Wesley Professional, MA: 1989.

[9] S. Baskar, P. Subberaj and M. V. C. Rao, "Hybrid Real Coded Genetic Algorithm Solution to Economic Dispatch Problem," Computers and Electrical Engineering, vol. 29, pp. 407-419, 2003.

[10] M. Srinivas and L. M. Patnaik, "Adaptive probabilities of crossover and mutation in genetic algorithms," IEEE Transaction on Systems Man and Cybermeties, vol. 24, pp. 656-667, 1994.

[11] T. Kuo and S. Huwang, "A genetic algorithm with disruptive selection," IEEE Transaction on Systems Man and Cybernetics-Part B: Cybernetics, vol. 26, pp. 299-307, 1996. 